\title{
用XPS 振起伴峰表征聚苯乙烯的辐射交联度
}

\author{
张万喜孙家珍 \\ (中国科学院长春应用化学研究所) \\ 陈传正岳 进 \\ (吉林化工研究院, 吉林)
}

人们知道 X-射线光电子能谱 (XPS) 振起 (Shake up) 伴峰主要是伴随主光电子的电离 所导致的最高占有轨道 (HOMO) 到最低末占有轨道 (LUMO) 电子䀓迁的结果. Carlson ${ }^{[1]}$ 及 $\mathrm{Clark}^{[2]}$ 等人均指出这种振起伴峰是某些过渡金属元素和共轭 $\pi$ 电子体系的表征. 聚苯乙 烯分子链上带有苯环侧基, 由于苯环上的共轭电子, 使得人们能够观察到这种伴峰. 这种伴峰 是由于 $\pi \rightarrow \pi^{*}$ 电子跃迁的结果, 它发生在苯环上, 其强度和 $\pi$ 电子共轭情况有关.

聚苯乙烯在高能射线的作用下将产生交联,而交联将影响苯环上的 $\boldsymbol{x}$ 电子共轭，进而影响 振起伴峰的强度亦即振起几率. 为此, 本文研究了不同辐射交联聚苯乙烯的 XPS 的振起几 率. 而这种振起几率在实验上是由伴峰与主峰的强度比来表征的. 并以此来表征交联聚苯乙 烯的交联度. 目前, 有关交联聚苯乙烯的 XPS 的振起伴峰的研究, 特别是用它表征交联度还 未见文献报道.

\section{实验}

试样的制备和辐照 将聚苯乙烯粒料在油压机上于 $140^{\circ} \mathrm{C} \sim 150^{\circ} \mathrm{C}, 100$ 公斤/厘米医力 下压制成约 0.2 毫米左右的薄膜. 在真空 $\left(2 \times 10^{-2}\right.$ 乇)下于常温用 60000 居里钴源 $\boldsymbol{r}$-射线辐 照.

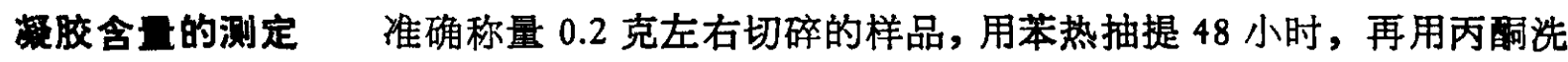
涤, 烘干恒重称量.

XPS 测试 用身津 ESCA-650B 型 X-射线光电子能谱仪, 配有 ESCAPAC-660 计算 机和曲线解析器, 以 $\mathrm{MgK}_{a 1.2} \mathrm{X}$-射线 $\left(E_{x}-1253.6 \mathrm{eV}\right)$ 作擞发源, 分析器室压力为 $8 \times 10^{-8}$ 乇, 对 $\mathrm{Au} 4 f 7 / 2$ 峰的半高宽为 $1.2 \mathrm{eV}$, 谱仪本身已对光电子动能 $\left(E_{k}\right)$ 做了校正,数字电压表 的读数即为光电子结合能 $\left(E_{b}\right)$, 以样品镀金的 $\mathrm{Au} 477 / 2$ 为 $83.8 \mathrm{eV}$ 做结合能校准.

\section{结果和讨论}

图 1 示出作为交联的和未交联的聚苯乙烯的 $\mathrm{C}_{\mathrm{s}}$ 光电子峰及其振起伴峰结构. 由图可见, 除在 $284.9 \mathrm{eV}$ 处的主峰外, 在高结合能端 $291.4 \mathrm{eV}$ 处出现振起伴峰结构, 其伴峰相对强度(振

本文 1984 年 5 月 25 日收到. 


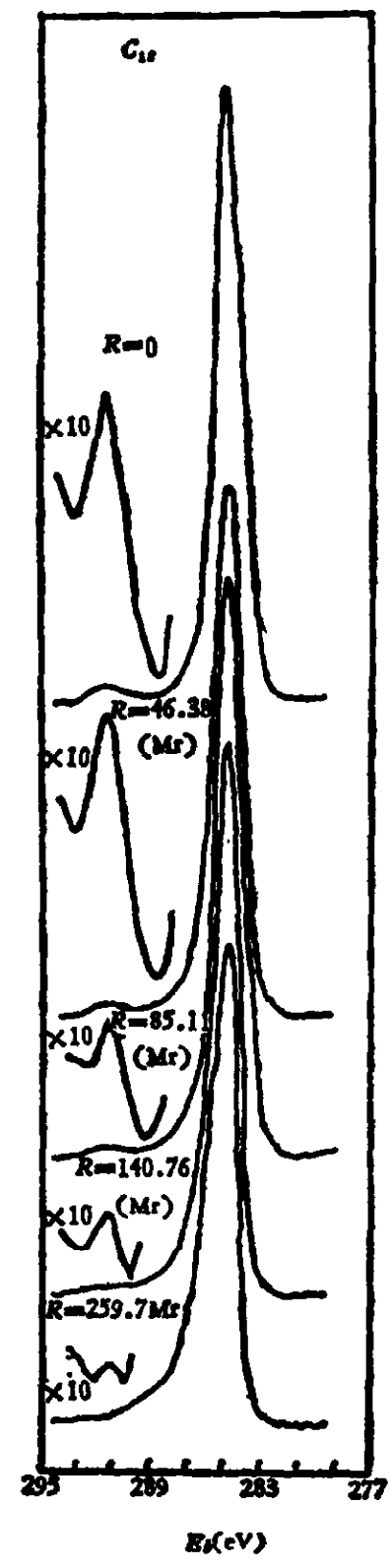

因 1 不同辐照剂是䈋苯乙 栟的 ESCA 谱
起峰面积 $/ \mathrm{C}_{1 s}$ 主蜂面积)即振起几率 $\left(P_{s}\right)$ 随辐照剂是 $(R)$ 的增加 而减小，这表明交联削弱了体系的 $\pi$ 电子共轭。我们认为，交联 产物的 $x$ 电子共轮下降的原因是和交联产物的结构有关，而产物 的结构是与交联机理有关。许多研究者 ${ }^{[3]}$ 做了大量的 ESR 研究, 似乎肯定聚苯乙焍在辐照过程中产生如下两种不同的自由基：

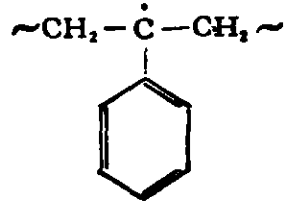

(1)

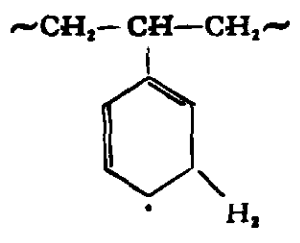

(II)
这两种自由基的偶合可能产生如下几种交联方式:

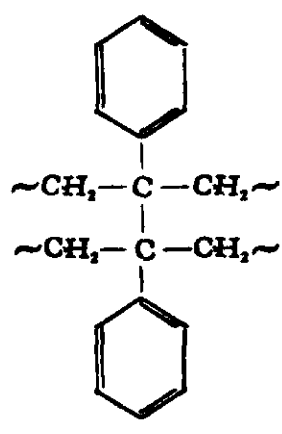

I + I

(i)

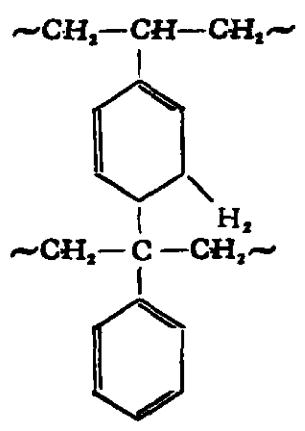

+ II

(ii)<smiles>CCC(C)C1=CCC(C2C=CC(C(CC)CC)=CC2C(CC)CC)C=C1</smiles>

II + II

(iii)

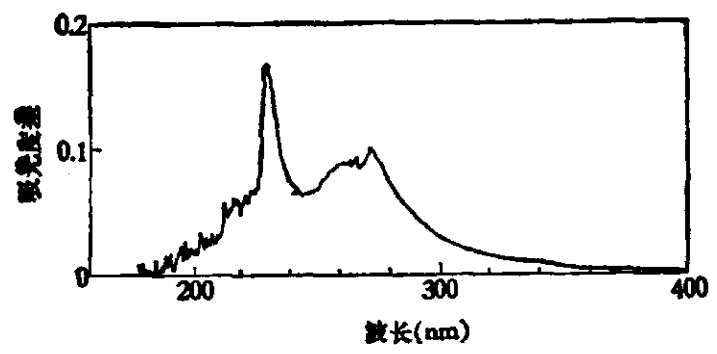

图 2 辐射交联聚苯乙烯的 UV 谱(以未辐照的聚莣乙烯为参比) $\mathrm{R}=293.8 \mathrm{Mr}$

第（i）种交联方式虽然苯环没有被破坏，但是由于交联后二个大分子的苯环相对位置会 发生变化, 这可能会使 $\pi$ 共轭效应下降, 由此造成振起几率下降. 许多研究者的实验结果也表 明有第 (ii) 种和第 (iii) 种交联的方式存在. Sears ${ }^{[\mathrm{l}]}$ 等人发现辐照后的聚苯乙烯的属于苯环 上的集团的红外吸收峰强度都有所减小, 这被认为是芳香性下降的证据. Slovokhotova ${ }^{[5]}$ 等人 研究了辐照后聚苯乙烯的紫外光谱, 并且指出有新的不具有芳香性的共轭不饱和集团形成.

我们以未交联的聚苯乙烯为参比, 做了辐射交联的聚苯乙烯的紫外吸收光谱(见图 2), 发 现辐照后的样品有新的吸收蜂出现,这也表明苯环在辐照过程中发生了变化.

由于观察到的振起几率主要是 $\pi$ 共轭体系中 $\pi \rightarrow \pi^{*}$ 跃迁的结果, 芳香性的破坏显然将 造成振起几率的下降. 所以，第 (ii) 种和第 (iii) 种交联方式必然导致产物的振起几率下降。 从而与其交联度相关. 为此我们用其振起几率来表征聚苯乙烯的交联度. 在凝胶点处, 其振 
起几率将开始发生变化. 图 3 给出了振起几率 $\left(P_{s}\right)$ 随剂量 $(R)$ 的变化关系. 由图中的曲线 形状我们可以看出在低剂量区域振起几率与剂量无关. 在其它剂量下, 则是指数关系. 以 $\log P$, 对 $\log R$ 做图, 我们可以得两条直线 (见图 4), 可以认为两直线相交处所对应的剂量就是 凝胶化剂量 $R_{\mathrm{gel}}$.

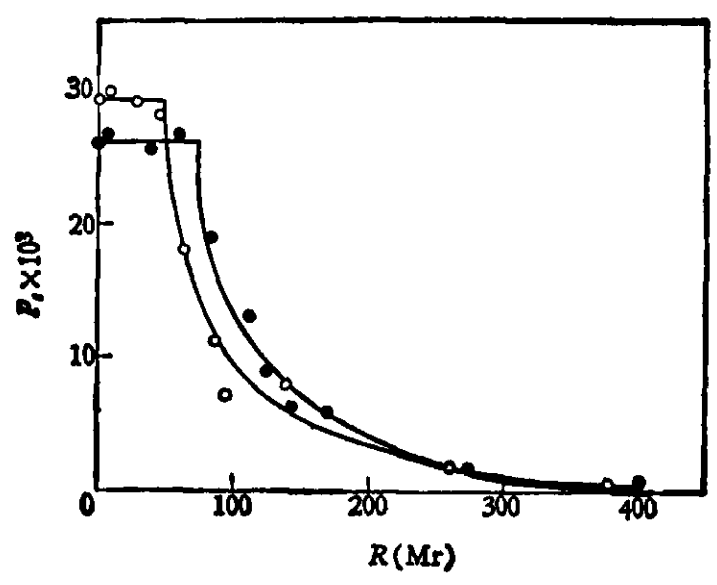

图 3 聚茉乙烯的 $P s$ 与 $R$ 的关系曲线 - $M_{* 0}=3.23 \times 10^{3} ; \quad 0 M_{\omega}=4.4 \times 10^{3}$

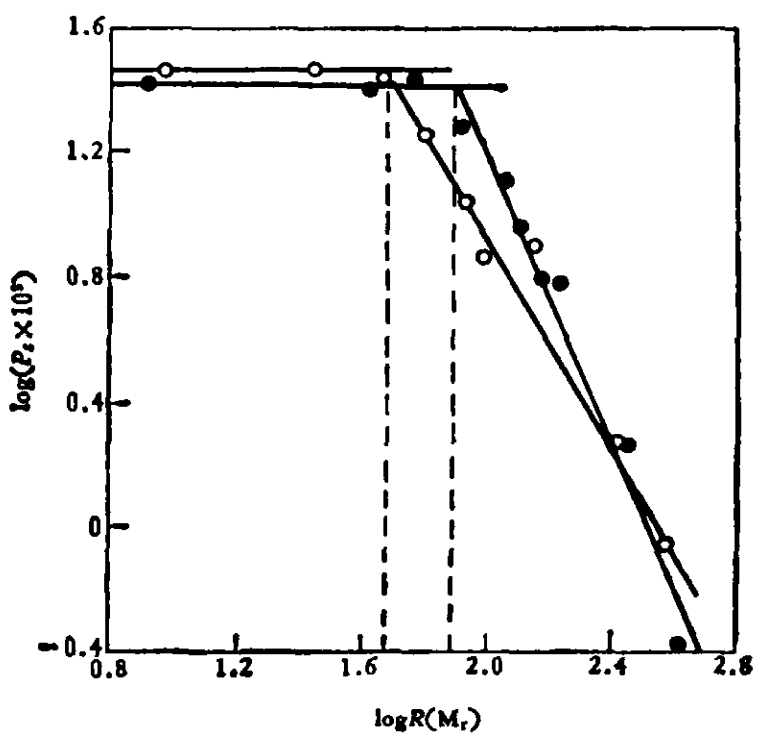

图 4 聚苯乙椧的 $\log P$ ，与 $\log R$ 的关系曲线

- $M_{w}=3.23 \times 10^{3} ; \quad \circ M_{w}=4.4 \times 10^{5}$

通常, 人们是按 Charlesby-Pinner 式 ${ }^{[6]}$

$$
s+\sqrt{s}=\frac{p_{0}}{q_{0}}+\frac{1}{q_{0} u_{1} R}
$$

来处理聚合物的辐射交联. 式中 $S$ 为溶胶分数, $P_{0}$ 和 $q_{0}$ 分别为单位剂量下的裂解和交联密 度, $u_{1}$ 为数均聚合度.

按此式以 $s+\sqrt{s}$ 与 $\frac{1}{R}$ 作图, 得一直线 (见图 5). 当 $s \rightarrow 1$ 时, 由直线斜率和截距可求 得凝胶化剂量。按上述两种方法求得的较胶化剂量和按下式 ${ }^{[7]}$ 计算出的交联 $G$ 值列在表 1 中.

$$
G=\frac{0.48 \times 10^{6}}{M_{w} \cdot R_{\mathrm{gel}}}
$$

$M_{w}$ 为重均分子量.

\begin{tabular}{|c|c|c|c|c|c|c|}
\hline \multirow{2}{*}{$M_{s o}$} & 凝 胶 & 含 & 法 & 振 & 几 & 事 法 \\
\hline & $R_{\mathrm{gel}}(\mathrm{Mr})$ & & G 值 & $R_{\mathrm{gel}}(\mathrm{Mr})$ & & G 值 \\
\hline $3.23 \times 10^{3}$ & 79 & & 0.019 & 79.4 & & 0.019 \\
\hline $4.4 \times 10^{3}$ & 52 & & 0.021 & 50.1 & & 0.022 \\
\hline
\end{tabular}

表 $1 \quad R_{\mathrm{gel}}$ 和 $G$ 值

由表 1 可知, 用振起几率方法求出的凝胶化剂量和 $G$ 值的数值与凝胶含量方法求出的相 一致. 从而这反过来确证本文用 XPS 振起几率来表征不同辐照剂量下聚苯乙烯交联度的合 理性. 


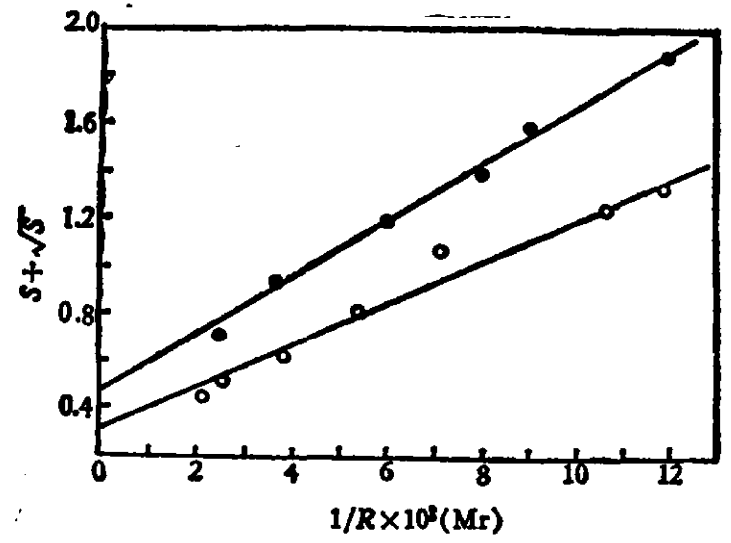

图 5 聚苯乙烯的 $s+\sqrt{ } \bar{S}$ 与 $1 / R$ 的关系曲线 - $M_{w}=3.23 \times 10^{3} ; \quad 0 M_{\infty}=4.4 \times 10^{3}$

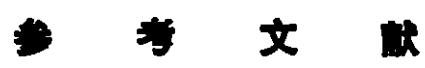

[1] Carlson, T. A. (王殿扐、郁向荣译), 光电于和俄能谓学,科学出版社, 1983.

[ 2 ] Clark, D. T. et al., J. Electron Spectrosc., 8(1976), 51.

[ 3 ] Dole, M. The Radiation Chemistry of Macromolecules, II(1973), 65-69.

[ 4 ] Sears, W. C. et al., Solid State Div. Ann. Progr. Rep., ORNL-2051, Nat. Tech. Info. Ser., Operations Div., Springfield, Virginia, 1956.

[ 5 ] Slovokhotova, N. A. et al., Vysokomol. Soedin, 3(1961), 191.

[ 6 ] Charlesby, A. and Pinner, S. H., Proc. Roy. Soc., A249(1959), 367.

[ 7 ] Charlesby, A., Atomic Radiation and Polymers, Pergamon Press, Oxford, London, New York, Paris, 1960. 